\title{
2-kW Average Power CW Phase-Conjugate Solid-State Laser
}

\author{
Yuri A. Zakharenkov, Todd O. Clatterbuck, Vladimir V. Shkunov, Alexander A. Betin, David M. Filgas, \\ Eric P. Ostby, Friedrich P. Strohkendl, David A. Rockwell, Member, IEEE, and Robert S. Baltimore
}

\begin{abstract}
We have demonstrated stable operation of a $2-\mathrm{kW}$ Yb:YAG phase-conjugate master oscillator power amplifier (PCMOPA) laser system with a loop phase-conjugate mirror (LPCM). This is the first demonstration of a continuous wave $(\mathrm{CW})$-input LPCM MOPA operating at a power greater than $1 \mathrm{~kW}$ with a nearly diffraction-limited output beam. The single-pass beam quality incident on the LPCM varied with the specific operating conditions, but it was typically $\sim 20$ times diffraction-limited (XDL). The measured beam quality with an MOPA output power of $1.65 \mathrm{~kW}$ was 1.3 XDL.
\end{abstract}

Index Terms-Beam quality, continuous wave (CW) high-energy laser, laser amplifiers, phase-conjugate mirror (PCM), power in the bucket, solid-state laser.

\section{INTRODUCTION}

$\mathbf{O}$ VER THE PAST 25 years, it has been clearly established that fairly simple devices based on nonlinear optical phase conjugation (NOPC) are capable of eliminating many problems arising from thermal loads in high-peak power pulsed solid-state lasers [1]-[3]. Present purposes can be served by simply stating that reciprocal phase aberrations induced in an optical beam by any medium can, in principle, be compensated by reflecting the aberrated beam off a phase-conjugate mirror (PCM) and passing the beam back through the aberrating medium. The output beam will have the same beam quality as the initial unaberrated beam.

More recently, the interest in high-power solid-state lasers has broadened to include new applications requiring continuous wave $(\mathrm{CW})$ or quasi-CW operation. Single-rod [4] and multipledisk [5] configurations have been reported with output powers $>1 \mathrm{~kW}$ (but with significantly reduced power for operation with $\left.M^{2}<10\right)$. Two-rod [6] and slab [7] lasers operating at greater than $400 \mathrm{~W}$ have also been described. Finally, the "heat capacity laser" [8] reached record power of $31.3 \mathrm{~kW}$, but only for $1-2 \mathrm{~s}$ of continuous operation, while operation for much longer times [9] was reported at $19 \mathrm{~kW}$.

These CW or quasi-CW systems are not very compatible with stimulated Brillouin scattering (SBS) phase conjugation (which

Manuscript received December 5, 2006; revised March 21, 2007. This work was supported in part by High Energy Laser Joint Technology Office and in part by Space and Airborne Systems, Raytheon, El Segundo, CA 90245.

Y. A. Zakharenkov, T. O. Clatterbuck, V. V. Shkunov, D. M. Filgas, F. P. Strohkendl, D. A. Rockwell, and R. S. Baltimore are with Raytheon Space and Airborne Systems, El Segundo, CA 90245 USA (e-mail: yuri_a_zakharenkov@raytheon.com).

E. P. Ostby is with Raytheon Space and Airborne Systems, El Segundo, CA 90245 USA and also with California Institute of Technology, Pasadena, CA 91125 USA.

A. A. Betin was with Raytheon Space and Airborne Systems, Raytheon, El Segundo, CA 90245 USA. He is now with the General Atomics, San Diego, CA 92121 USA.

Digital Object Identifier 10.1109/JSTQE.2007.896565 has been utilized in the vast majority of pulsed phase-conjugate (PC) solid-state lasers) for two main reasons. First, their output powers are much lower than the peak powers achieved by even modest pulsed solid-state lasers, making it difficult to reach the SBS threshold. Second, even very low absorption coefficients in the nonlinear medium can produce unacceptably high thermal loads at required operational power levels.

In view of these limitations to the applicability of SBS phase conjugation, we began developing an alternative NOPC architecture approximately 10 years ago. This alternative architecture is called a loop phase-conjugate mirror (LPCM), and this name is derived from the fact that the nonlinear medium is incorporated into a loop resonator [10]. Our LPCM exploits a thermal nonlinearity, whereby four-wave mixing (FWM) occurs in an absorbing medium. The physical basis of the FWM is the fact that the presence of two interfering high-power laser beams (a signal beam and a reference beam) in the absorbing medium produces a spatially varying temperature distribution, and consequently, a spatially varying refractive index or hologram in the nonlinear medium. This hologram can be "read" by a third beam, thereby producing PC replica of the input signal beam.

This LPCM offers several important advantages over SBS in high-average power laser applications. First, an LPCM can function over a wide peak- and average-power dynamic range. This allows operation at power levels ranging from low values of a few watts to high values of many kilowatts. Second, an LPCM offers broad wavelength coverage, and finally, it is compatible with lasers producing short coherence lengths ( $\sim 1 \mathrm{~cm}$ or less). Several low-power experimental LPCM demonstrations have been reported [10], [11]; these demonstrations are consistent with the advantages asserted earlier. However, to our knowledge, there have been no prior reports of LPCMs that might be used in high-power $\mathrm{CW}$ phase-conjugate master oscillator power amplifier (PC-MOPA) laser systems.

This paper represents the first successful demonstration of a quasi-CW solid state PC-MOPA laser system producing a nearly diffraction-limited beam with power $>1 \mathrm{~kW}$. Section II describes the overall system architecture, while Section III describes the LPCM, and Section IV summarizes our experimental results.

\section{SYSTEM ARCHITECTURE}

Fig. 1 shows a schematic diagram of our double-pass PCMOPA. The signal beam originates from a commercial $\mathrm{Yb}: \mathrm{YAG}$ master oscillator [12]. The master oscillator (MO) output passes through a Faraday isolator and an outcoupler into the amplifier 


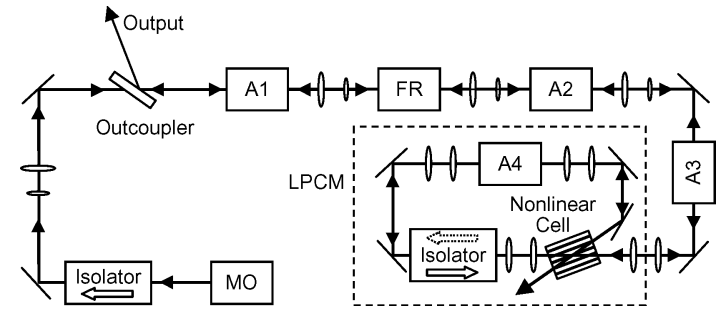

Fig. 1. Schematic diagram of the PC MOPA system.

beamline that comprises one or more Yb:YAG amplifier slabs (A1-A3).

Relay-imaging telescopes are an important element of the MOPA system, and they are used throughout the amplifier chain and also within the LPCM. In addition, high-power variable apertures are located at the foci of the relay optics to function as spatial filters. Each of these imaging telescopes is schematically shown as a single pair of lenses in the figure, but in some cases, a telescope can also contain cylindrical optics (not shown) that might be used to change the aspect ratio of the beam.

The imaging optics and their spatial-filtering capability play an important function in the MOPA system. First, the optics reimage the pupil from one stage to the next, ensuring that the beam exiting one amplifier is delivered to the next amplifier with high efficiency. Consequently, during the initial signal pass through the amplifiers, the imaging telescopes avoid any significant loss of aberration information at the hard apertures that are distributed along the beam path. Such loss of information would degrade the possible phase conjugation fidelity.

Second, during both passes through the amplifier chain, the spatial-filtering capability prevents a large fraction of the highly divergent amplified spontaneous emission (ASE) from entering successive amplification stages and extracting power from the amplifiers or causing a parasitic oscillation.

The amplifier transverse dimensions correspond to an aspect ratio of $18: 1$, which approximately matches the far-field divergence $\left(10^{\circ} \times 0.6^{\circ}\right)$ of typical fast-axis-collimated laser diode arrays. This allows coupling the output from a 2-D laser diode array into the slab with a single lens. At the required power levels, the thermal lensing in the thin (fast) axis of a slab is very high if light would be traveling along the axis of the slab. The amplifiers employ zigzag signal propagation, which has long been known to mitigate the effects of fast-axis thermal lensing in face-cooled laser slabs.

The high aspect ratio amplifier slabs induce only slight depolarization during operation. Hence, we can achieve the outcoupling function by inserting a Faraday rotator (FR) into the amplifier/LPCM path (for example, between A1 and A2, as shown in the figure). This rotator is double-passed by the signal beam. Consequently, the second-pass output polarization is orthogonal to that of the first-pass beam. A common polarizing beam splitter then separates the MOPA output beam from the orthogonally polarized oscillator input beam.

The LPCM is also schematically shown in Fig. 1. The aberrated first-pass beam, denoted as beam 1, enters the LPCM and passes through the cell that contains the nonlinear medium.

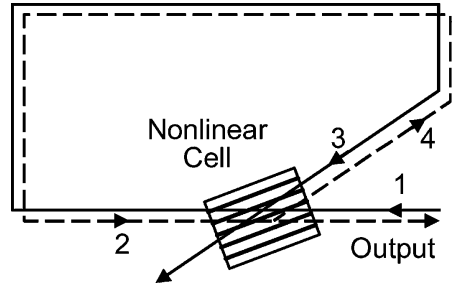

Fig. 2. Schematic diagram of nonlinear cell (NC) indicating labels on the four interacting beams.

Following the nonlinear cell (NC) is a Faraday isolator favoring propagation in the counterclockwise direction, but that is slightly detuned to allow a few percent of the initial beam to pass through in the clockwise direction toward the LPCM amplifier A4. This amplifier raises the power of the beam, now denoted as beam 3, to the same level as beam 1 . This attenuation of beam 1 is important to ensure that beam 1 does not extract too much power from A4-system efficiency is greatly enhanced if the counterpropagating beam extracts most of the power from the amplifiers. Beams 1 and 3 are made to overlap in the nonlinear medium, where the interference between the beams produces a spatially varying refractive index pattern (i.e., a hologram) in the nonlinear medium.

This hologram provides the feedback necessary to initiate oscillation in the counterclockwise direction for the loop laser resonator, which is formed by the amplifier A4, the hologram as a resonator mirror, and several folding mirrors, as shown in Fig. 1. The beam generated by this laser oscillation is denoted as beam 2 in the vicinity of the $\mathrm{NC}$ and as beam 4 just after it is diffracted back into the loop by the dynamic hologram of the NC (see Fig. 2). Under a wide range of operating conditions, the loop output beam 2 is the conjugate of the loop input beam 1. Most of beam 2 passes through the NC to form the LPCM output, thereby functioning as the conjugate seed that initiates the second pass through the amplifier chain.

\section{LPCM}

The basic operation of an LPCM has been described earlier [10], [11], and a detailed description of the present LPCM is also available [13]. The key components of the LPCM are the NC, the LPCM amplifier A4, and the isolator (see Fig. 1). Diffraction by the hologram grating in the $\mathrm{NC}$ allows it to function simultaneously as a resonator mirror, a transverse-mode selector, and an outcoupler. For typical operating conditions, the hologram diffraction efficiency is designed to be low, approximately a few percent, so, the $Q$-factor of the ring-laser resonator is low. The LPCM amplifier provides the gain required to compensate the resonator losses at the outcoupler and the other optical elements. It also provides power for the PC output beam. The isolator prohibits ring-laser oscillations in the forward (clockwise) direction, preserving the optical power for the backward (counter-clockwise) PC beam.

A variety of nonlinear processes can be used in the LPCM NC. In this paper, we employ a thermal nonlinearity, and the nonlinear medium is an absorbing liquid. The hologram arises from 
the fact that the spatially varying intensity distribution produced by the interference of beams 1 and 3 (the two beams incident from the right-hand side in Fig. 2) induces a spatially varying temperature distribution in the absorbing liquid, which leads to a spatially varying index distribution through the temperature dependence of the refractive index.

The choice of an optimum liquid is essential and is based on the trade study reported in [14]. Consider the situation in which a pair of plane waves propagating in an absorbing medium forms a refractive index grating having a spatial period $\Lambda$. The steady-state diffraction efficiency $\eta$ for such a thermal grating follows from the model presented in [14], [15], and for the approximation of weak diffraction, the diffraction efficiency is given by

$$
\eta=(\alpha L)^{2}\left[\left(\frac{\Lambda^{2}}{2 \pi \lambda}\right)^{2}\right]^{2}\left(\left(\frac{d n}{d T}\right) / \chi\right)^{2} I_{1} I_{3}
$$

where $\alpha$ is the liquid absorption coefficient at the signal wavelength, $L$ is the thickness of the absorbing layer in the direction of the beam propagation, $\lambda$ is the signal wavelength, and $I_{1}$ and $I_{3}$ are intensities of the writing beams. The first term in (1) combined with the final terms (the beam intensities) represent the total power absorbed in the medium, while the second term (in square brackets) implicitly embodies the system geometry (beam-crossing angle) within the grating spatial period. The third term indicates how the refractive index responds to the intensity grating formed by the two writing beams, including the time dependence of the thermal gratings that is governed by the thermal diffusivity of the nonlinear medium, $\chi$. Equation (1) shows that, for a given absorption, the two key material parameters are $(d n / d T)$ and $\chi$. These parameters drive the material selection. Additional medium-selection parameters include a sufficiently high boiling temperature, a low chemical reactivity, and preferably a minimal health hazard. Typical candidate nonlinear media include acetone, toluene, carbon tetrachloride, benzene, and carbon disulfide.

We have developed a model to predict the LPCM performance under typical operating conditions. The model assumes that, for a broad range of extracted optical powers $P_{\text {ext }}$, the loop amplifier gain $G$, follows exponential saturation $G=G_{0} \exp \left(-P_{\text {ext }} / P_{\text {sat }}\right)$, characterized by a saturation power $P_{\text {sat }}$. The model further assumes that the grating reflectivity $\eta$ obeys (1), specifically that it is proportional to the product of the powers of two writing beams as $\eta=\nu P_{1} P_{3}$. The effective parameters for the loop amplifier-the small signal gain $G_{0}$ and the saturation parameter $P_{\text {sat }}$-depend on the diode pump power and the input beam alignment. The slope $\nu$ for the grating efficiency is controlled by the sizes of the writing beams as well as the cell geometry and operating conditions.

By simply invoking power balance per round-trip for both propagation directions, one can derive explicit relations for the output power $P_{2}$ and a threshold $P_{\text {thr }}$ with respect to the input power $P_{1}$; for the limit of interest, corresponding to a low grating efficiency $\eta \ll 1$, these expressions can be simplified to

$$
P_{2} \approx P_{\mathrm{sat}} T \ln \left(\frac{P_{1}}{P_{\mathrm{thr}}}\right), \quad P_{\mathrm{thr}}=\frac{\exp \left(\sqrt{\tau_{F} / \nu P_{\mathrm{sat}}^{2}}\right)}{G_{0} T \sqrt{\tau_{F} \nu}}
$$

where $T$ is the loop round-trip transmission and $\tau_{F}$ is the Faraday transmission of beam 1 in the forward (clockwise) direction. The loop reflectivity reaches a maximum for an input signal power $P_{1}=e P_{\mathrm{thr}} \approx 2.71 P_{\mathrm{thr}}$, yielding a reflected power $P_{2}^{\max }=T P_{\text {sat }}$, which is determined by only two parametersthe amplifier saturation power and the linear round-trip loss. Experiments demonstrate excellent agreement between the predictions of (2) and measured data [13].

So far, this discussion has focused on the energetics of the LPCM. However, given that the function of the LPCM is to achieve phase conjugation, it is appropriate to consider how the loop resonator can be optimized such that the dominant transverse mode of the resonator is the PC replica of the input signal beam. Detailed studies indicate that the grating in the $\mathrm{NC}$ possesses a fine-structured fringe pattern that preferentially reflects the PC portion of beam 2 back into the loop resonator, thereby allowing the PC mode to have the lowest threshold. Several measures can be taken to discriminate in favor of the PC mode against noise components [16]-[20] including reducing the size of beam 3 relative to beam 1 and inserting a spatial filter that efficiently transmits the input signal beam but blocks much of the spatial noise.

\section{EXPERIMENTAL RESULTS}

The fully integrated PC-MOPA system was operated for extended time periods $\sim 30 \mathrm{~min}$ or more, exhibiting stable outputbeam parameters and excellent beam quality. Fig. 3 shows the calculated and measured far-field intensity distributions in the focal plane of a 530-mm lens while the PC-MOPA system was producing $\sim 1.65-\mathrm{kW}$ output power. The corresponding gains and extracted powers of the individual power amplifiers are summarized in Table I.

During these measurements, the near-field intensity distribution approximately filled a rectangular aperture having dimensions of $5.5 \times 4.5 \mathrm{~mm}^{2}$. The left-hand side of Fig. 3 indicates the calculated far-field intensity distribution for such a near-field aperture, assuming a uniform near-field intensity and planar phase over the entire aperture. The measured farfield intensity distribution is also shown for comparison, and it is obvious that the two distributions are very similar. This is quantified in the power-in-the-bucket (PIB) curves shown in Fig. 3. The blue curve represents the calculated PIB curve for the uniform intensity/phase distribution, while the red curve represents the experimental measurements. At the $50 \%$ power level, the experimental curve is $\sim 1.3 \mathrm{XDL}$. Note that far-field power measurements through a series of hard apertures show that $>90 \%$ of the total PC-MOPA output power is contained within the field of view of the video camera and analysis system that was used to generate the far-field data in Fig. 3. 

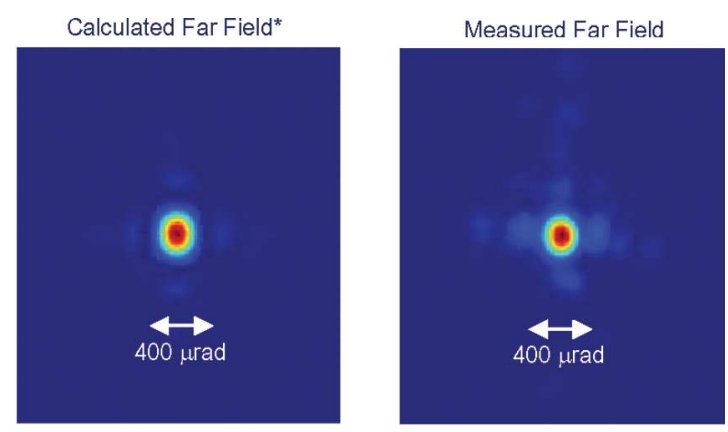

*Uniform Near Field, $5.5 \times 4.5 \mathrm{~mm}^{2}$

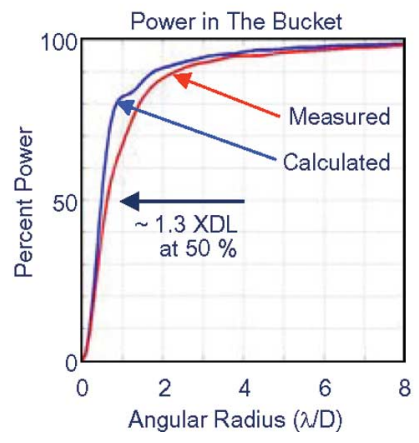

Fig. 3. Calculated and measured far-field intensity profiles at the focal plane of a lens with an effective focal length of $530 \mathrm{~mm}$. Calculation assumes a uniform intensity and phase over an entire $5.5 \times 4.5 \mathrm{~mm}^{2}$ aperture. Power-in-the-bucket curve shows that the measured PC MOPA output beam quality is $\sim 1.3 \mathrm{XDL}$ at the $50 \%$ power level. The beam size, or $D$ value, used in the calculated PIB is $5 \mathrm{~mm}$.

TABLE I

Gain ANd Total Double-PASS EXTRACTEd POWER From THE NONOPTIMIZED YB:YAG POWER AMPLIFIERS WHILE OPERATING THE OVERALL SYSTEM AT 1.65-KW OUTPUT POWER

\begin{tabular}{lll}
\hline \hline Amplifier & Gain & Extracted Power, W \\
\hline A1 & 4 & 1300 \\
A2 & 3.9 & 345 \\
A3 & 7 & 235 \\
& & \\
\hline \hline
\end{tabular}

Subsequent data were taken at higher powers. Fig. 4 shows a temporal record of the MOPA output power $\sim 2 \mathrm{~kW}$ over a time period of approximately $10 \mathrm{~min}$.

In addition to the overall system, we quantified the performance of the LPCM [13]. It suffices here to note that the experimental characterization of the two nonlinear components of the operating LPCM - the thermal cell and the saturated amplifier - both performed as expected, based on the aforementioned model.

We compared the performance of the overall PC-MOPA system with the predictions of a model that uses measured characteristics for each element and the transmission losses between elements to calculate the power for both the first and second pass, between each pair of amplifiers, and at the final output beam. Each power amplifier was represented by an exponential dependence of the gain $G$ on extracted power $P_{\text {ex }}$ as given by $G=G_{0} \exp \left(-P_{\mathrm{ex}} / P_{\text {sat }}\right)$, using measured values for the small-

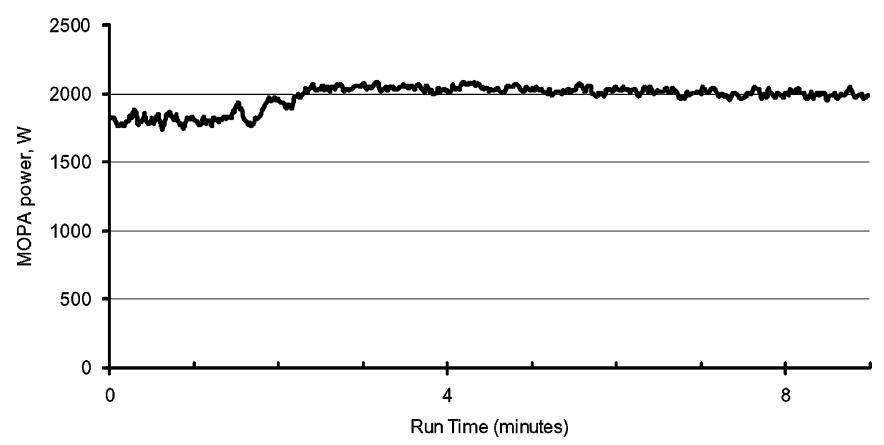

Fig. 4. PC MOPA operation with output power exceeding $2 \mathrm{~kW}$.

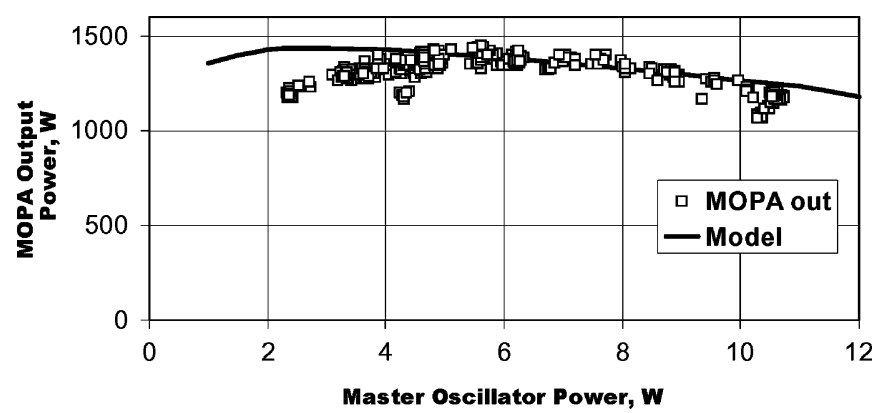

Fig. 5. Experimental points and model (curve) results for MOPA output power versus master oscillator input power.

signal gain $G_{0}$ and the saturation power $P_{\text {sat }}$. Although this gain-saturation equation is identical to the one discussed earlier in connection with the LPCM amplifier, the quantitative values of the parameters $G_{0}$ and $P_{\text {sat }}$ are different, as they are also for the various power amplifiers. This approximation provides a good match with the empirical data for limited intervals of extraction, and it is consistent with a more rigorous standard saturation model for the gain media. The functional dependence of the reflected power for the LPCM on its operating conditions was derived from a separate model that matched all of our LPCM data rather well.

Fig. 5 compares the PC-MOPA model with experimental results, where the overall output power is plotted against the input power $E_{0}$ of the master oscillator light incident on the outcoupler. For these experiments, $E_{0}$ was varied by a factor of 5 with relatively minor changes in overall system performance. For these calculations, the polarization outcoupler transmission is taken as $95 \%$ both ways, and the transmission between the amplifiers is taken as $80 \%$ for the first pass and $90 \%$ for the second pass; the first and second pass spatial filter transmissions between A3 and the LPCM were taken as $75 \%$ and $100 \%$, respectively.

\section{CONCLUSION}

We have generated up to $2 \mathrm{~kW}$ of quasi-CW average laser power with a nearly diffraction-limited beam quality using a double-pass PC-MOPA architecture incorporating an LPCM. The output beam quality was quite good, and we present data 
showing a beam quality of $\sim 1.3 \mathrm{XDL}$. The output power achieved in this research was primarily limited by the performance of the power amplifiers and not by the LPCM. Hence, we believe scaling to significantly higher powers $\sim 25 \mathrm{~kW}$ or more will be possible with improved amplifiers.

\section{ACKNOWLEDGMENT}

The authors would like to thank N. P. Davis, J. J. Ichkhan, R. G. Hegg, T. Matsuoka, S. McGanty, R. A. Reeder, D. Reinard, S. Sorbel, R. Zamudio, and R. Zhou for their excellent support.

\section{REFERENCES}

[1] V. V. Shkunov and B. Ya. Zel'dovich, "Optical phase conjugation," Sci. Amer., vol. 253, pp. 54-59, Dec. 1985.

[2] D. A. Rockwell, "A review of phase-conjugate solid-state lasers," IEEE J. Quantum Electron., vol. 24, no. 6, pp. 1124-1140, Jun. 1988.

[3] D. M. Pepper, D. A. Rockwell, and G. J. Dunning, "Nonlinear optic phase conjugation," IEEE Circuits Devices Mag., vol. 7, no. 5, pp. 21-34, Sep. 1991.

[4] H. Bruesselbach and D. S. Sumida, "A 2.65-kW Yb:YAG single-rod laser," IEEE J. Sel. Topics Quantum Electron., vol. 11, no. 3, pp. 600-603, May/Jun. 2005.

[5] C. Stewen, M. Larionov, A. Giesen, and K. Contag, "Yb:YAG thin disk laser with $1 \mathrm{~kW}$ output power," Adv. Solid-State Lasers: OSA Trends in Opt. and Photonics, vol. 34, H. Injeyan, U. Keller, and C. Marshal, Eds. Washington, DC: Opt. Soc. Amer., 2000, pp. 35-41.

[6] E. C. Honea, R. J. Beach, S. C. Mitchell et al., "High-power dual-rod Yb:YAG laser," Opt. Lett., vol. 25, no. 11, pp. 805-807, 2000.

[7] G. D. Goodno, S. Palese, J. Harkenrider, and H. Injeyan, "Yb:YAG power oscillator with high brightness and linear polarization," Opt. Lett., vol. 26, no. 21, pp. 1672-1674, 2001.

[8] M. D. Rotter, C. B. Dane, S. Fochs et al., "Solid-state heat-capacity laser: Good candidates for the marketplace," Photon. Spectr., vol. 38, no. 8, pp. 44-56, 2004.

[9] G. D. Goodno, H. Komine, and S. J. McNaught et al., "Coherent combination of high-power zigzag slab lasers," Opt. Lett., vol. 31, no. 9, pp. $1247-1249,2006$

[10] A. A. Betin, S. C. Matthews, and M. S. Mangir, "Phase conjugation of depolarized light with a loop PCM," in Proc. Conf. Nonlinear Opt. '98: Mater., Fundam. Appl. Top. Meet., Aug. 1998, pp. 288-290.

[11] A. A. Betin, R. W. Byren, and R. S. Baltimore et al., "High power solidstate lasers with phase conjugation," presented at the Phys. Quantum Electron. (PQE) Winter Colloq., Snowbird, UT, Jan. 2002.

[12] Electronic Laser System Corp., Fort Worth, TX, 2006. [Online]. Available: http://www.versadisk.com

[13] A. A. Betin, V.V. Shkunov, and Y.A. Zakharenkov et al., "200 W phaseconjugate mirror for $\mathrm{CW}$ radiation," submitted for publication.

[14] O. L. Antipov, A. A. Betin, E. A. Zhukov, and S. G. Turgenev, "Four-wave interaction of middle-infrared radiation in media with thermal nonlinearity," Sov. J. Quantum Electron., vol. 19, no. 11, pp. 1465-1473, 1989.

[15] B. Y. Zel'dovich, N. F. Pilipetsky, and V. V. Shkunov, Principles of Phase Conjugation. Berlin, Germany: Springer-Verlag, 1985.

[16] A. A. Betin, "Phase conjugation based on thermal nonlinearity," in Proc. Conf. Nonlinear Opt. '96: Mater., Fundam. Appl. Top. Meet., Maui, HI, Jul.1996, pp. 336-339.

[17] A. A. Betin and N. Y. Rusov, "On the formation of a conjugate wave in a scheme of four-wave mixing with feedback," Izv J. Vyssh.Uchebn. Zaved, Radiofizika, vol. 30, p. 767, 1987.

[18] A. A. Betin and N. Y. Rusov, "Structure of lasing modes generated as a result of a four-wave mixing with a feedback," Sov. J. Quantum Electron., vol. 18, p. 657, 1988.

[19] V. I. Bespalov and A. A. Betin, "Spatial structure of radiation from lasers with controllable nonlinear mirrors," Bull. Acad. Sci. USSR Phys. Series, vol. 53 , no. 8 , pp. 52-59, 1989 .

[20] A. A. Betin and A. V. Kirsanov, "Selection of phase conjugate wave in an oscillator based on a four-wave interaction with feedback in an extended nonlinear mirror," Sov. J. Quantum Electron., vol. 24, no. 3, pp. 219-222, 1994.

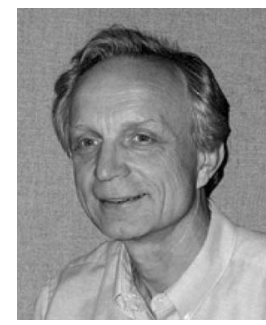

Yuri A. Zakharenkov was born in Moscow, Russia, on April 9, 1948. He received the M.Sc. degree in physics from Moscow State University, Moscow, in 1972, and the Ph.D. degree in quantum radiophysics from Lebedev Physical Institute, Moscow, in 1978.

During 1972-1991, he was a Senior Research Associate and a Group Leader at the Lebedev Physical Institute, Moscow. During 1991-1992, he investigated laser-produced plasma at Laser Facility Vulcan, Rutherford Appleton Laboratory, Oxford, U.K. From 1993 to 1999, he was an Associate Researcher at Short Pulse Laser Facility, Lawrence Livermore National Laboratory, University of California, where he was engaged in laser beam characterization. He has designed and assembled focusing optics and plasma diagnostics for 100-TW laser and investigated laser beam propagation through powerful high-aperture laser amplifier. During 1999-2002, he was a Team Leader in telecom industry, developing novel high-speed all-optical transceiver module for fiber optical communication network. He joined Raytheon Space and Airborne Systems, El Segundo, CA, as a Senior Principal Physics Engineer in November 2002 to take part in high-power laser programs. He is the holder of two U.S. patents. He has Authored/Coauthored two monographs on laser-produced plasma diagnostics, 65 publications in the field of lasers and nonlinear optics, and over 30 reports in published conference proceedings.

Dr. Zakharenkov is a member of the Optical Society of America

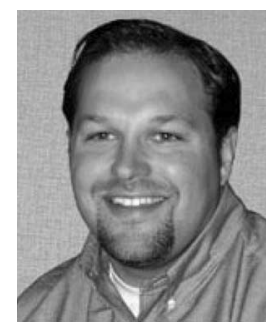

Todd O. Clatterbuck was born in Columbus, $\mathrm{OH}$ in 1973. He received the B.A. degree in physics from Wittenberg University, Springfield, OH, in 1996, and the $\mathrm{Ph} . \mathrm{D}$. degree in atomic physics from the State University of New York (SUNY), Stony Brook, in 2003.

$\mathrm{He}$ is currently with the Raytheon Space and Airborne Systems, El Segundo, CA, where he performs advanced development work in diode-pumped solidstate laser systems. He has developed a number of continuous-wave kilowatt-class laser systems based mostly on diode-pumped Yb:YAG. His current research interests include defense applications of diode-pumped ultrashort laser systems. He has Authored/Coauthored numerous papers published in journal and conference proceedings.

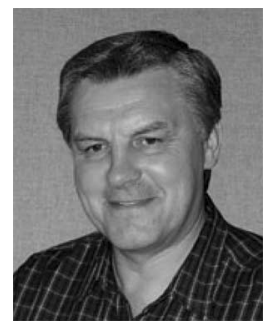

Vladimir V. Shkunov was born in Moscow, Russia, on July 11, 1953. He received the M.S. and Ph.D. degrees in physics from Moscow Institute of Physics and Technology, Dolgoprudny, Russia, in 1977 and 1979 , respectively.

During 1979-1997, he was a Junior Research Associate, and then a Team Leader in the Institute for Problems in Mechanics, Russian Academy of Sciences, Moscow, studying basic aspects of nonlinear optical phase conjugation, speckles, and dynamic holographic gratings. During 1997-2000, he worked on photorefractive optics and RF photonics as a Visiting Fellow, and then as a Research Associate at JILA, University of Colorado, Boulder. From 2000 to 2003, he was a Senior Physicist in Trans Photonics, Chicago, IL, where he contributed to developing direct writing techniques for polymer-based integrated optics. In 2003, he joined the High-Energy Laser Team, Raytheon Space and Airborne Systems, El Segundo, CA, as a Senior Principal Physics Engineer. He has coauthored three monographs on phase conjugation and laser speckles, and has published over 120 papers in the field of nonlinear optics and lasers.

Dr. Shkunov is a member of the Optical Society of America. 


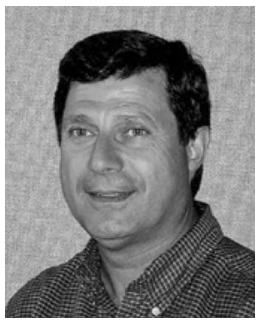

Alexander A. Betin was born in Nizhny Novgorod, Russia, in 1950. He received the M.S. and Ph.D. degrees in electronics from the Lobachevsky University, Nizhny Novgorod, in 1972 and 1978, respectively.

During 1972-1994, he worked as a Senior Research Scientist and the Head of Laser Physics and Nonlinear Optics Laboratory, Institute of Applied Physics, Russian Academy of Science, Nizhny Novgorod. From 1994 to 1999 , he was a Senior Research Scientist, Hughes Research Laboratories, Malibu, CA. In 1999, he joined Raytheon Space and Airborne Systems, El Segundo, CA, where he was a Senior Engineering Fellow and Technical Director for high-power laser development programs. Since 2006, he has been a Chief Laser Scientist at Photonics Division, General Atomics, San Diego, CA. He is the holder of 44 granted and pending patents. He is the author or coauthor of more than 60 journal papers and book chapters, 90 conference presentations, and invited talks. His current research interests include nonlinear optics, phase conjugation, and gas and solid state high-power lasers.

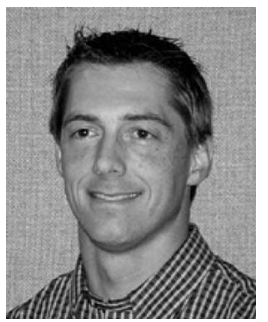

David M. Filgas received the B.S. and M.S. degrees in electrical engineering from Stanford University, Palo Alto, in 1989 and 1990, respectively.

He has developed multikilowatt solid-state laser systems. In January 2002, he joined Raytheon Space and Airborne Systems, El Segundo, CA, where is actively involved in the development of highpower diode-pumped solid-state lasers for sensing and high-energy laser (HEL) weapon applications. Planar waveguide amplifier technology developed at Raytheon by him currently holds the world record for power extraction from a single laser crystal at $16 \mathrm{~kW}$ and shows the potential for scalability to HEL power levels in a single amplifier. He has also adapted the planar waveguide architecture to compact, robust, $100-\mathrm{W}$ average power, nanosecond pulse systems for sensing applications. As a Development Director for the Joint High Power Solid-State Laser (JHPSSL) Program, he guided the development of multikilowatt Yb:YAG slab amplifiers and phase conjugation technology used in Raytheon's phase-conjugated master oscillator power amplifier (PC-MOPA) architecture. Before joining Raytheon, he has developed four generations of multikilowatt solid state fiber-delivered industrial lasers for GSI Lumonics. He holds nine U.S. patents. He has Authored or Coauthored numerous papers published in journals and conference proceedings.

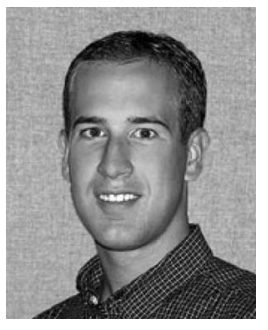

Eric P. Ostby received the B.S. degree in electrical engineering from the University of Minnesota, Minneapolis, in 2002, and the M.S. degree in electrical engineering from the California Institute of Technology, Pasadena, in 2004, where he is currently working toward the Ph.D. degree.

He completed an internship at IBM, where he worked on the laser and optical receiver systems of a $10-\mathrm{GB} / \mathrm{s}$ optical transceiver product. He has demonstrated a spatial-mode-division multiplexing optical fiber system using holographic techniques.

At Raytheon Space and Airborne Systems, El Segundo, CA, he has developed a nanosecond pulse multiple kilowatt peak-power master oscillator and has worked with the high-energy laser team. Also, he has achieved record results for ceramic microchip lasers. He has Authored or Coauthored several conference proceedings papers.

Mr. Ostby is a member of the Optical Society of America (OSA) and is currently the President of the OSA Student Chapter at the California Institute of Technology.

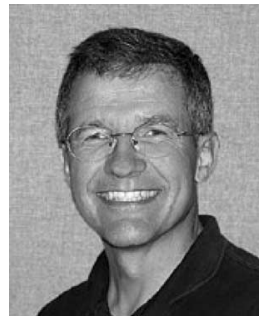

Friedrich P. Strohkendl was born in Wurzburg, Germany, on March 10, 1956. He received the M.Sc. degree in physics from the University of Wurzburg, Wurzburg, Germany, in 1981, and the Ph.D. degree in physics from the University of Southern California, Los Angeles, in 1988.

In 1989, he produced the first low-loss waveguides in $\mathrm{KNbO}_{3}$ at the Swiss Federal Institute of Technology. In 1990, he devised highly effective oxidation/reduction treatments for altering the photorefractive properties of $\mathrm{BaTiO}_{3}$ at Hughes Research Laboratories. During 1991-2000, he established a frequency agile femtosecond laboratory for the study of ultrafast optical nonlinearities in organic materials at the University of Southern California. He has developed a pulsed amplifier with record low-energy fluctuation of $0.4 \% \mathrm{rms}$, which enabled the measurement of the first femtosecond degenerate and nondegenerate four-wave mixing spectra. From 2000 to 2004, he developed low-loss fiber optic collimator arrays for telecom applications and invented methods for amplified spontaneous emission (ASE) suppression in high-gain systems in the field of high-pulse energy fiber lasers. In June 2004, he joined Raytheon Space and Airborne Systems, El Segundo, CA, as a Senior Principal Physics Engineer, where he is engaged in high-power solid-state lasers. He holds one U.S. patent. He has reviewed 19 publications in the fields of lasers and nonlinear optics.

Dr. Strohkendl is a member of the Optical Society of America.

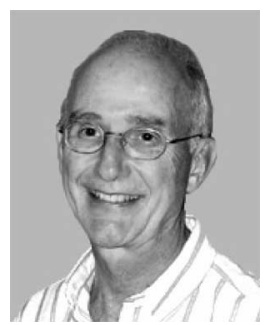

David A. Rockwell (M'90) was born in Chicago, IL, on November 11, 1945. He received the B.S. degree in engineering physics from the University of Illinois, Urbana, in 1967, and the Ph.D. degree in physics from the Massachusetts Institute of Technology, Cambridge, in 1973.

He was a Postdoctoral Research Fellow in the Department of Physics and Electrical Engineering, University of Southern California, Los Angeles, where he was engaged in radiative absorption at the surfaces of high-power infrared laser window materials. In 1975, he joined the Hughes Aircraft Company, Culver City, CA, where he was engaged in the development of advanced solid-state lasers and nonlinear optical devices. In 1981, he joined the the Advanced Laser Sources Department, Hughes Research Laboratories (HRL), Malibu, CA, where he was a Principal Research Scientist and Manager and was involved in the early work of nonlinear optical phase conjugation and the application of this technology to high-power solid-state lasers. From 1995 to 1997, he was on special assignment for HRL, Delco Electronics Manufacturing Technology Center, Kokomo, IN, where he has developed a number of manufacturing applications of lasers and optics. In 1997, he became a Senior Scientist and Process Champion for optical communications at Hughes Space and Communications Company, El Segundo, CA, where he worked on laser sources for optical intersatellite laser communications. In 2000, he became the Director of Advanced Technology at fSONA Communications, Manhattan Beach, CA, where he was responsible for establishing and leading a program of strategic research and development for commercial free-space optical communications. Since 2002, he has been with business unit of Raytheon Space and Airborne Systems, El Segundo. His research has been concerned with the development of high-power solid state and fiber lasers. He has 26 publications in refereed journals and more than 60 publications in conference presentations, tutorials, and invited talks.

Dr. Rockwell is a member of the Optical Society of America. 


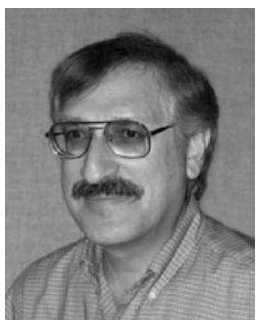

Robert S. Baltimore received the B.S.E.E. degree from the Northeastern University, Boston, MA, and the MBA degree from Pepperdine University, Malibu, CA.

He has managed the High Energy Laser (HEL) Development Team, which executed the HEL Joint Technology Office phase conjugate master oscillator power amplifier (PC MOPA) Key Technologies Contract, Laser Engineering and Technology Support Kilowatt Level Fiber Laser and Beam Combination Contract, UTC Multi-Jet Impingement Cooling of Solid State Slab Laser Contract, and numerous Raytheon-funded programs supporting the science and technology for high-energy/power laser system. Before that, he was engaged in the slab laser technology development for the U.S. Army's multifunction laser and the very successful product development for the U.S. Army's eyesafe laser rangefinder (ELRF) for the LRAS3. He is a Systems Engineering Manager with Raytheon, where he has collaborated in the development of Element 6 Laser Doppler Velocimeter, Airborne Laser Proposal, and ELRF Raman-shifted product line, which includes the Bradley ELRF (BELRF), Elite I and II, M1, and improved target acquisition system (ITAS) ELRF. He was involved in the Advanced Test and Manufacturing Technology for Raytheon's (legacy Hughes) Production Engineering Department/Laboratory, which produced many innovative production techniques that are still in use today. His research has been concerned with the development of laser systems beginning with the M60A3 laser rangefinder. 